\title{
Glatiramer acetate in the treatment of multiple sclerosis
}

\author{
Alex Tselis \\ Omar Khan \\ Robert P Lisak \\ Division of Neuroimmunology, \\ Department of Neurology, Wayne \\ State University School of Medicine, \\ Detroit, MI, USA
}

\begin{abstract}
Glatiramer acetate is an immunomodulating drug used in the treatment of multiple sclerosis. It consists of a copolymer of amino acid residues in the same stoichiometric proportions as in myelin basic protein. Its mechanism of action is not entirely known and is probably multifaceted, with deletion of some immune cell populations and stimulation of others in these patients. Some mechanisms involve neuroprotectant effects. There is ample evidence of its efficacy in relapsing-remitting disease, using both clinical and imaging measures of disease activity, and in this paper we review the clinical and basic studies of this drug. Finally we discuss how some of its neuroprotectant effects may be useful in neurodegeneration such as is seen in more advanced cases of multiple sclerosis and other diseases such as amyotrophic lateral sclerosis and Parkinson's disease.
\end{abstract}

Keywords: glatiramer acetate, multiple sclerosis, neurodegeneration

\section{Introduction}

Multiple sclerosis (MS) is an inflammatory demyelinating disease of the central nervous system (CNS), affecting separate regions of the brain and spinal cord at different times, with axonal damage. It is usually relapsing and remitting (RRMS), at least initially. The disease often enters a secondary progressive (SPMS) phase, with smoothly increasing deficits, leading to substantial disability over time. In this phase of the disease, the pathogenesis is more "degenerative" than inflammatory. It must be acknowledged that this is an oversimplification and depends on defining the inflammatory phase of RRMS as gadolinium enhancement of lesions on magnetic resonance imaging (MRI) T1 sequences, a marker of changes in the blood-brain barrier to humoral factors. Thus the lack of gadolinium enhancement would not rule out infiltration of inflammatory cells and it is known that using triple dose of gadolinium shows that many lesions thought not to enhance with a single dose still have blood-brain barrier defects. In addition the persistence of cerebrospinal fluid (CSF) oligoclonal bands and the detection of inflammatory cells in the brain and spinal cord of SPMS patients, in the absence of gadolinium enhancement, further supports a persistence of the inflammatory component in the pathogenesis of MS lesions even during the "degenerative" phase of the course of the disease. A small proportion of cases are progressive at onset, and this is known as the primary progressive form of MS (PPMS). The term "chronic progressive" MS (CPMS) is an obsolete term encompassing SPMS, PPMS, and progressive relapsing MS (PRMS). More recently it has become clear that there are extensive changes in the gray matter including cerebral cortex and deep nuclear structures such as the thalamus and that these changes occur very early in the course of the disease. The mechanisms of these neuronal changes are not well understood and there are likely several of importance. Moreover the relationships between the pathogenic processes in white 
matter with those seen in the gray matter are not clear (Kidd et al 1999; Peterson et al 2001; Kutzelnigg et al 2005)

Glatiramer acetate (GA) is an immunomodulating agent approved by the FDA for the treatment of RRMS. The drug was originally prepared as an analog of myelin basic protein (MBP), and consists of a random copolymer of L-alanine, L-lysine, L-glutamic acid, and L-tyrosine, in a molar ratio 4.2:3.4:1.4:1.0, respectively, as a string of 40-100 amino acid residues. The compound was used in research on experimental allergic encephalomyelitis (EAE), an animal model of MS, in which the injection of myelin antigens (whether purified in adjuvant or crude extracts of white matter) triggers inflammatory demyelination, with some parallels to MS. The drug was found to strongly inhibit the inflammatory demyelination which occurs in EAE (Arnon 1996).

\section{Clinical studies and trials Open label studies}

The success of GA in preventing and ameliorating EAE suggested its potential for benefit in MS, and preliminary studies were initiated early on.

In the first published report of the use of GA in humans, Abramsky et al (1977) gave GA to 3 patients with acute disseminated encephalomyelitis (ADEM) (2 mg intramuscularly daily) and 4 patients with severe MS (2-3 mg intramuscularly every 2-3 days). The drug was well tolerated.

An open label study of 12 patients with CPMS and 4 with RRMS examined the effect of GA given intramuscularly, originally intended to be given at decreasing doses over a 6-month period. However, there were hints of efficacy and many of the patients continued the drug at doses of up to $20 \mathrm{mg}$ a day, for 18 months to more than 2 years. None of the patients deteriorated and a few seemed to improve (Bornstein et al 1981).

\section{Controlled trials}

In an important pilot trial of GA, 50 RRMS patients were divided into treatment and placebo groups, with patients individually matched for gender, relapse frequency and degree of disability before entering the study (Bornstein et al 1987). The degree of disability was measured by the Kurtzke extended disability status score (EDSS) (Kurtzke 1983). The treatment group received GA $20 \mathrm{mg}$ daily for 2 years. The primary outcome variable was the proportion of relapse-free patients, while secondary outcomes consisted of frequency of exacerbations, change in disability and time free of progression. The results showed clear benefit with GA. The proportions of relapse-free patients in the treatment and placebo groups were $56 \%$ and $26 \%(\mathrm{p}=0.045)$ respectively.
The number of relapses in the treatment and placebo groups was 16 and 62. Interestingly, the less disabled patients had more benefit in the relapse rate than the more disabled patients, in the treatment group, suggesting a greater efficacy the earlier treatment is started (Tselis and Khan 2004).

The next trial examined the efficacy of GA in CPMS. In this study 106 "chronic progressive" patients, at 2 sites (Albert Einstein, Bronx, NY and Baylor, Houston, Texas), were randomized to either placebo or GA $15 \mathrm{mg}$ twice daily for 2 years (Bornstein et al 1991). The primary endpoint was the proportion of patients with confirmed progression. Confirmed progression was defined as a change in EDSS by 1.0 or 1.5 steps (for those with baseline EDSS greater or less than 5.0 respectively) sustained for 3 months. As previously stated, "chronic progressive" MS is not a homogeneous entity and included both SPMS and PPMS, which dilutes the patient groups and makes valid comparisons difficult.

Overall, there was no statistically significant (in the sense of $\mathrm{p} \leq 0.05$ ) difference in the primary outcome between the two groups at the 24 month time point. Interestingly, there was a trend (in the sense of $0.05 \leq \mathrm{p} \leq 0.10$ ) to benefit from GA at both the 12- and 24-month time points, with progression in $20.4 \%$ of treatment and $29.5 \%$ of placebo subjects at two years, $p=0.086$ (Bornstein et al 1991). When the results were analyzed by center, however, some interesting observations were made. At the Einstein site there was a statistically significant beneficial effect, with a probability of progression of $21.4 \%$ in treated patients and $38.5 \%$ in placebo patients, $\mathrm{p}=0.041$. At Baylor there was no difference, with $19 \%$ of both treated and placebo patients progressing. The placebo patients at Einstein had a much higher rate of progression (with 34\% progressing) than the Baylor patients (only $14.8 \%$ progressing). The reason for this is unknown, but may have to do with the relative proportions of SPMS and PPMS patients at each center.

The pivotal trial, leading to the approval of GA by the Food and Drug Administration (FDA) for RRMS, consisted of 251 patients randomized to GA $20 \mathrm{mg}$ subcutaneously once a day or placebo, for 24 months (Johnson et al 1995). The patients had EDSS scores between 0 and 5.0, at least 2 relapses within the preceding 2 years, and no recent corticosteroid or immunomodulatory therapy. The primary endpoint was the mean number of relapses per patient during 2 years of treatment, with a comparison between the treatment and placebo patients. A number of secondary outcomes were studied, including proportion of patients relapse-free, time to first relapse after initiation of therapy and proportion of 
patients with progression (defined as a one step increase in the EDSS sustained for at least 3 months).

The results showed a statistically significant decrease in relapse frequency, a trend to benefit in delay to the next relapse and proportion of patients relapse-free and no statistically significant effect on progression-free proportion. Thus, the GA group had 161 relapses and the placebo group 210 , giving an average number of relapses in 2 years of 1.19 and 1.68 , respectively, with $p=0.0007$. The median time to first relapse after enrollment was 287 and 198 days in the treatment and placebo groups $(\mathrm{p}=0.097)$. The proportion of patients relapse-free was $33.6 \%$ and $27.0 \%$ in the treatment and placebo groups, respectively $(\mathrm{p}=0.098)$. The proportion of patients progression-free was $78.4 \%$ and $75.4 \%$ in the treatment and placebo groups, respectively (NS).

The drug was well tolerated, causing insignificant injection site reactions. In about $15 \%$ of glatiramer patients and $3 \%$ of placebo patients, a peculiar reaction occurred just after injection. This consisted of some combination of flushing, chest tightness, dyspnea, palpitations, or anxiety, lasting a few seconds to half an hour, and resolved without any residual problem. The reaction usually occurred only once, but one patient had seven episodes (Johnson et al 1995).

\section{The extension study}

In order to continue to collect data on the effect of GA on the disease, the pivotal study was extended by up to 11 months, with the GA versus placebo arms intact and blinded allowing all patients to reach the 2-year time point (Johnson et al 1998). Of the 215 patients completing the original study, 203 entered the extension study and 194 completed it. The primary and secondary outcomes were the same. The relapse rates over the extended study were 1.34 and 1.98 for the GA and placebo groups, respectively $(\mathrm{p}=0.002)$. The relapsefree proportions were 33.6 and $24.6(\mathrm{p}=0.035)$, and the median times to first relapse were 287 and $198(\mathrm{p}=0.057)$ for the GA and placebo groups, respectively. Overall status was defined by change in EDSS, with "improvement" or "worsening" defined as a one step decrease or increase in EDSS. While most patients had no change in EDSS over the extended study, some improved and others worsened. In the GA more improved than worsened (27.2\% vs $18.4 \%)$, while in the placebo group, fewer improved than worsened (12.0\% vs 31.2).

\section{The open-label extension study}

At the end of the extension study, all patients were invited to continue follow-up in an open-label study on GA, so that further clinical data could be collected. Most of the patients in the original double blind study, 208 out of 251 originally randomized (83\%), chose to continue into the open label study, which is still ongoing. At the 8-year time point, 142 patients ( $57 \%$ of the originally randomized and $68 \%$ of those who entered the open label phase) were still being followed (Johnson et al 2005). In order to analyze the effect of early treatment on MS, the patients were divided into two groups: group A were patients who had received GA from the beginning and group $B$ patients were initially randomized into the placebo group and subsequently were on open label GA, and their relapse rates and degree of disability ascertained. At the 8-year time point of the open label study, the annualized relapse rates were 0.43 in the group A and 0.52 in the group B patients, $p=0.0459$. The overall proportion of patients with stable or improved neurological status (as measured by the EDSS) was $65.3 \%$ in group A and $50.4 \%$ in group $B(p=0.0263)$.

\section{Imaging studies}

The European/Canadian (E/C) Imaging Study examined the effect of GA on MRI disease activity (Comi et al 2001), which was only done for a short period of time on patients at one of the 11 centers in the original pivotal study. In the E/C study, 239 RRMS patients were randomized to GA or placebo, and had monthly MRI scans and neurological examinations. The primary outcome was the number of enhancing lesions on T1 weighted images. There were a number of secondary outcome variables, including other measures of imaging activity and clinical events. The total number of enhancing lesions per subject over the 9 months of the study was 33.7 in the placebo group and 21.8 in the GA group $(\mathrm{p}<0.001)$. The difference between the groups became statistically significant 5 months into the study. The relapse rate in the treated group ( 0.51 per subject) was less than that in the placebo group ( 0.76 per subject), by $33 \%$ $(\mathrm{p}=0.012)$. Other imaging outcomes were favorable to GA. In particular, the $\mathrm{T} 2$ lesion burden, as roughly measured by the mean total number of new T2 lesions, was 13.5 in the placebo and 9.4 in the active treatment groups, a $30 \%$ difference $(p<0.003)$.

In a substudy of the E/C Imaging Study, the proportion of initial T1-hypointense lesions evolving into permanent black holes, which represent permanently damaged tissue, was compared between the GA and placebo groups (Filippi et al 2001). Of all T1-hypointense lesions, at 8 months, $15.6 \%$ in the GA group and $31.4 \%$ in the placebo group had evolved into black holes, thus suggesting a protective effect of GA 
$(\mathrm{p}<0.002)$. There was also a reduction in re-enhancing lesions by $59 \%$ when comparing the treated patients with those in the placebo group.

Patients in this trial were recruited into an open-label extension phase lasting another 9 months, with the placebo subjects getting open label GA and the treatment subjects continuing on their medication (Wolinsky et al 2002). It was found that the number of enhancing lesions decreased by $54 \%$ in those who went from placebo to GA, and further decreased by $24.6 \%$ in those who continued on GA from the doubleblind to the open label phase (Wolinsky et al 2002). Interestingly, the difference in T2 lesion burden persisted over the 18 months of the study, suggesting that early treatment may preserve tissue, which was not regained in the switch from placebo to open label (Wolinsky et al 2002).

Imaging studies have been used to detect the extent of "neurodegeneration" by various surrogate measures. One possibility would be to use brain atrophy as a surrogate for the effects of degeneration. In a substudy of the original pivotal GA study, 27 patients were randomized to GA or placebo, and the number of enhancing T1 and T2 lesions, as well as brain parenchymal volume measured over 2 years. There was less atrophy in the GA group than in the placebo group (Ge et al 2000). In another study comparing two different methods of measuring brain atrophy, using data from the E-C study, there was a significant decrease in the development of brain atrophy in the GA group compared with the placebo group, when using the more sensitive measure (Sormani et al 2004).

Magnetic resonance spectroscopy (MRS) measurements of brain chemistry have been very useful in understanding the pathogenesis of neurological diseases, and MS has been extensively studied. Various markers of neuronal and myelin structure have been measured and correlated with pathology and disability. N-acetyl aspartate (NAA) is a marker of neuronal structure and axonal integrity. Its decrease in MS plaques indicates axonal injury, which may be only partly reversible (Arnold et al 2001). In a pilot study of the effect of GA on axonal injury in RRMS, 22 patients were recruited, 18 of whom were treated with GA and 4 of whom declined treatment because of aversion to needles (Khan et al 2005). The patients were followed for 2 years. MRS measured NAA in a volume of interest (VOI) containing abnormal signal as well as in normal-appearing white matter (NAWM). In the GA group, the NAA-to-creatinine ratio (NAA/Cr, easily measured for technical reasons) had increased in the VOI by $10.7 \%$ in the treated group, while it had decreased by $8.9 \%$ in the untreated group. Similarly, in the NAWM, the
NAA/Cr was increased by $7.1 \%$ in the treated group, while it decreased by $8.2 \%$ in the untreated group.

\section{Anti-glatiramer antibodies}

The issue of the generation of antibody to GA is an important one since antibodies can theoretically abrogate the beneficial effect of the drug, as seems to be the case for interferon-beta and natalizumab therapy. Antibodies to GA acetate have been detected in the sera of patients treated with the drug (Brenner et al 2001). In this study, sera from 217 patients who participated in the treatment and placebo arms of several GA acetate trials were tested for antibodies to GA using an ELISA method. Antibodies to GA were of the IgG (more IgG1 than IgG2) but not IgM or IgE isotypes. They were detected in all of the active treatment patients but not in the placebo patients. The titers of these antibodies increased to a maximum at 3 months after initiation and slowly decreased thereafter. There was no change in antibody titers to myelin basic protein. Another study showed the presence of IgG4 antibodies, detected by ELISA, in all 20 MS GA-treated patients, and in a minority of MS patients not treated with GA and normal controls (Farina et al 2002). There was no evidence of adverse effect of the antibodies, and indeed patients with higher antibody titers seemed to be more likely to be relapse-free (Brenner et al 2001). In vitro correlates of GA effect were observed either not affected or enhanced by antiGA antibodies in one study (Teitelbaum et al 2003) but not in another (Salama et al 2003). In the latter study it was proposed that some of the patients had antibodies that blocked the therapeutic effects but the in vitro immunologic endpoints were not necessarily measures of all of the mechanisms of action of GA, the clinical observations were not blinded and the number of patients was small.

\section{Glatiramer acetate in primary progressive MS}

A phase 3 trial of GA acetate in primary progressive MS (PPMS) was initiated in 1999 (Wolinsky 2004). The study randomized 943 patients to either GA or placebo for 36 months, with a placebo-controlled extension phase. The primary endpoint was progression of disease as ascertained by a sustained ( 3 month) increase in EDSS by 1 point for baseline EDSS 3.0-5.0 (low tier) and by 0.5 point for baseline EDSS 5.5-6.5 (high tier). Secondary endpoints included proportion of progression-free patients, changes in EDSS scores, number and volume of various lesions on MRI, black hole lesion burden, and brain volume loss. The study was discontinued early (at 
the second interim analysis) because the progression rate in the placebo group was unexpectedly small: it was assumed that $50 \%$ of the low tier EDSS patients would progress in a year, but only $16.1 \%$ of patients in the placebo group did so (Wolinsky 2004). This rendered it impractical to demonstrate a clear treatment effect on the primary endpoint. However, there was a trend to reduced progression, significantly decreased enhancing lesions and lower increases in T2 lesions in the treatment group compared with the placebo group (Wolinsky et al 2007). Thus, it may be premature to use glatiramer in PPMS, since the trial was unable to demonstrate benefit in the target population and the long term safety of the drug was not demonstrated in this group, unlike RRMS.

\section{Systematic reviews and the Cochrane analysis}

The efficacy of GA in MS was examined in two metaanalyses of clinical trials, one by the Cochrane Collaboration (Munari et al 2003) and the other by Boneschi et al (2003). In the Cochrane study, the results of three published studies were pooled (Bornstein et al 1987, 1991; Johnson et al 1995). In the Boneschi study, 3 studies, only one of which was in the Cochrane list, were analyzed together (Bornstein et al 1987; Johnson et al 1998; Comi et al 2001). The published studies were used in the Munari study, while the Boneschi study used the raw data from the original trials. The outcome variables examined in these two studies are shown in the Table 1.

The Munari study did not show an effect of GA on progression at 2 years, or change in disability score at follow up, but did show a trend to a decrease in the number

Table I Outcome variables examined in the Munari and Boneschi meta-analyses

\begin{tabular}{|c|c|}
\hline $\begin{array}{l}\text { Munari et al } \\
\text { Primary outcomes }\end{array}$ & $\begin{array}{l}\text { Boneschi et al } \\
\text { Primary outcome }\end{array}$ \\
\hline Progression at 2 years & Annualized relapse rate \\
\hline \multicolumn{2}{|l|}{$\begin{array}{l}\text { Change in disability at } \\
\text { follow up }\end{array}$} \\
\hline \multirow{3}{*}{$\begin{array}{l}\text { Number of patients with } \\
\text { at least one exacerbation } \\
\text { Relapse-free survival }\end{array}$} & Secondary outcomes \\
\hline & \\
\hline & On-trial total number of relapses \\
\hline Secondary outcomes & $\begin{array}{l}\text { Time to first relapse } \\
\text { Accumulated disability }\end{array}$ \\
\hline \multicolumn{2}{|l|}{$\begin{array}{l}\text { Hospitalizations at end } \\
\text { of follow up }\end{array}$} \\
\hline \multicolumn{2}{|l|}{$\begin{array}{l}\text { Steroid courses at end } \\
\text { of follow up }\end{array}$} \\
\hline \multicolumn{2}{|l|}{ Additional outcomes } \\
\hline Mean number of relapses & \\
\hline
\end{tabular}

of patients with at least one exacerbation when a stratified analysis was done, but not when the results were pooled. Relapse-free survival was better in the GA group, as were the number of hospitalizations and steroid courses at the end of follow up. The mean number of relapses showed no significant changes in years 1 and 2, but a decrease in year 3 in the GA group (Munari et al 2003). The study was criticized for using summary data rather than raw data, using results that mixed relapsing-remitting MS and "chronic progressive" MS patients (these latter being a mixture of primary and secondary progressive MS), redefinition of some of the outcome variables (confirmed progression being defined at 1 year rather than the more conventional 3 or 6 months), and choice of analysis (Caramanos and Arnold 2005; Comi et al 2005). In two of the studies incorporated in the Munari study, relapse numbers were measured. In the Bornstein study (Bornstein et al 1987), the number of relapses was 16 in the GA group and 64 in the placebo group, while in the Johnson study (Johnson et al 1995) the number of relapse per patient in 2 years was 1.19 in the GA group and 1.68 in the placebo group, a clear difference in favor of GA in both studies. No such benefit was seen in the combined groups, however, which seems counterintuitive (Comi et al 2005).

The Boneschi meta-analysis showed significant decreases in the annualized relapse rate, on-trial total number of relapses, time to first relapse, and accumulated disability, favoring the GA group (Boneschi et al 2003). The different studies incorporated in the analysis had patients with a uniform diagnosis of relapsing-remitting MS but different baseline ages, disease durations, baseline EDSS, prestudy relapse rate, which were adjusted for.

\section{Glatiramer mechanisms of action including neuroprotection models}

Given the complexity of GA it is not surprising that there are likely several mechanisms of action. It was one of a number of compounds originally synthesized to help understand EAE, an animal model of MS. EAE was first discovered by Rivers and Schwentker in an investigation of the inflammatory demyelination in the brain which occurred after rabies vaccination and some viral infections such as measles (Rivers and Schwentker 1935). These compounds contained polypeptides consisting of amino acids with stoichiometry similar to that of myelin basic protein, and were expected to provide well-defined antigens with which to induce EAE (Arnon 1996). It turned out that none of these compounds caused EAE in animals, and in fact, several inhibited it; copolymer 1 (the original name for GA) did this most 
effectively. This provided a rationale for studying its effect on MS, as described above.

Several potential mechanisms of action have been suggested. These are not mutually exclusive, and all probably contribute to the drug's efficacy. Given the molecular resemblance of some of the components of the drug to fragments of myelin basic protein, it is likely that molecular mimicry (in some sense) drives the effects of the drug on the disease process.

The postulated steps in the immune reaction to myelin are as follows, in a very oversimplified version. An activated $\mathrm{T}$ cell, sensitive to a myelin antigen (such as myelin basic protein (MBP), myelin oligodendrocyte glycoprotein (MOG), myelin associated glycoprotein (MAG) or others) penetrates the blood-brain barrier, and encounters an antigen-presenting cell (APC). The APC presents a peptide fragment from the relevant myelin antigen (perhaps in the process of turnover of myelin in the central nervous system), nestled in the groove of the major histocompatibility class II molecule (MHC-II), to the $\mathrm{T}$ cell, along with costimulatory ligands. The $\mathrm{T}$ cell receptor engages the peptide fragment nestled in the $\mathrm{MHC}$ II and the appropriate costimulatory ligands on the antigen presenting cell (APC) bind to the coreceptors on the T cell. The APC directs the phenotype of the T cells that are generated, with M1-type APCs stimulating T cells in a T helper-1 (Th1) or "proinflammatory" configuration and M2-type APCs promoting Th2 or "anti-inflammatory" T cells. In MS, the APCs seem to adopt an M1-phenotype, elaborating IL-12 which activates the $\mathrm{T}$ cell into a Th1 state with production of Th1-type cytokines (such as IL-2, IFN- $\gamma$, TNF $\alpha$ ). TNF $\alpha$ is also elaborated by APCs. These cytokines open up the bloodbrain barrier and allow ingress of other cellular and humoral inflammatory mediators into the brain, eventually resulting in demyelination and injury to axons. There is strong evidence that in both MS and EAE Ig synthesis and deposition with complement activation contribute to demyelination (Raine et al 1999; Lucchinetti et al 2000).

The GA molecule most likely substitutes for the myelin peptide fragment in the APC and exerts its effects from there. The GA molecule is known to inhibit the proliferative $T$ cell response to MBP (Teitelbaum et al 1992). The potential mechanisms of action include the following.

(1) The GA molecule binds to the MHC-molecule on the APC so strongly that it displaces fragments of MBP from the APC which would otherwise have been presented to an appropriately sensitized $\mathrm{T}$ cell (Fridkis-Hareli et al 1994). This binding occurs equally well with different MHC-II molecules, indicating a certain "promiscuity" in the choice of MHC-II haplotype to be used by GA (Fridkis-Hareli et al 1994). This may be one possible explanation of why GA prevents EAE induced by PLP in mice, although it is not known that GA displaces PLP from the MHC-II molecule (Teitelbaum et al 1996). It is not clear how relevant this mechanism of MBP antigen displacement is, however, since presumably APCs loaded with MBP are present in the brain, and subcutaneously injected GA will not have easy access to them.

(2) The GA-MHC-II complex binds to the T cell receptor of the $\mathrm{T}$ cell, thus preventing its activation by the MBPMHC-II complex on the APC. This would cause blockade of T cell receptors by a GA-MHC-II complex competing with an MBP-MHC-II complex. This may not happen centrally, since access of subcutaneously injected GA, complexing with an MHC-II molecule, does not easily have access to APCs located centrally. However, such an effect has been demonstrated in vitro (Aharoni et al 1999).

(3) The GA-MHC-II complex binds to the T cell receptor and partially activates the $\mathrm{T}$ cell, inducing tolerance by an "altered peptide ligand" mechanism (Aharoni et al 1999). GA thus induces a deviation from a pro-inflammatory Th-1 immune response to an anti-inflammatory $\mathrm{T}$ helper-2 (Th-2) response, with secretion of the cytokines IL-4, IL-5, IL-10, IL-13, and TGF- $\beta$, which some investigators consider a Th3 cytokine. Lymphocytes secreting anti-inflammatory factors would explain the clinical improvement in animals with EAE who still have inflammatory lesions, with the presence of lymphocytes, after treatment (Aharoni et al 2000; Lisak et al 1983). The quantity of anti-inflammatory cytokines induced by the cross-reactivity of MBP and GA seems to be at the level of inducing increased cytokine secretion on a per cell basis (Farina et al 2001) rather than by induction of proliferation of lymphocytes. (Burns and Littlefield 1991; Lisak et al 1983). This may be an alternative mechanism for how GA ameliorates PLP-induced EAE. That is, GA induces MBP reactive Th2 cells to release the same protective cytokines which in turn downregulate the response of PLP-reactive Th1 cells. This has been called "bystander protection". The therapeutic implications of this are evident (see below).

In EAE, Th-1 cells are thought to be mainly responsible for the disease state, since the disease can be transmitted to naïve syngeneic recipients by transfer of Th-1 cells, and resolution of disease is associated with emergence of Th- 2 cells. By analogy, Th-1 cells are thought to have a pathogenic role 
in MS. GA has been shown to induce a deviation from a "proinflammatory" Th1 state in T cells to an "anti-inflammatory" Th2 state, both in vitro and in vivo. Thus, when dendritic cells (DC), which are highly efficient APC, are treated with GA, secretion of IL-12, a cytokine which causes Th-1 differentiation of naïve Th cells, decreases in a dose-response manner. Naiive Th cells exposed to GA-treated DCs thus differentiate into Th-2 cells (Vieira et al 2003). EAE is suppressed in mice treated with Th-2 GA-induced T cells (Aharoni et al 1997). In a study of $8 \mathrm{MS}$ patients treated with GA, GA-specific T cells were deviated to a Th-2 polarization (Qin et al 2000). This has been seen in other studies ( Duda et al 2000; Neuhaus et al 2000; Chen et al 2001).

There are other mechanisms which may also contribute to the therapeutic effect of GA in MS. As stated above, APCs activate antigen-specific T cells of either a Th1 or Th2 phenotype, depending on whether the APC has an M1 or M2 phenotype (Kalinski et al 1999). It has been shown that GA influences APC towards an M1 (IL-12 secreting) or M2 (IL-10 secreting) phenotype (Hussien et al 2001; Farina et al 2005; Kim et al 2004; Weber et al 2004) which may in turn influence the resultant proportion of Th1 and Th2 lymphocytes reactive to GA and cross reacting myelin antigen specific T cells. There are reports of changes in function of monocytes/macrophages/microglia and dendritic cells in patients treated with GA (Balabanov et al 2001; Hussien et al 2001; Weber et al 2004)

The lymphocytes of normal individuals and GA naive patients with MS respond to GA in vitro with a vigorous proliferative response whereas patients treated with GA lose this response. The loss of a proliferative response seems to be due to both clonal deletion and clonal anergy (Ragheb et al 2001). This would reduce the number of potentially myelin reactive cells that also recognize GA which would be of potential benefit. Finally there is evidence that GA also can bind to MHC class 1 but not CD and thus influence CD8 (cytotoxic and immunosuppressive) T cells (Karandikar et al 2002). Finally, as mentioned above, GA-specific T cells may have a neuroprotectant action by elaborating neurotrophic factors in addition to anti-inflammatory cytokines. Activated T cells specific for myelin antigens have been shown to produce brain-derived neurotrophic factor (BDNF) (Kerschensteiner et al 1999) In a study of T cell lines obtained from GA-treated MS patients, $14 \%$ of the GA-reactive T cells (all Th-2) and smaller proportions of MBP- and tetanus toxoid (TT)-reactive $\mathrm{T}$ cells were found to secrete BDNF (Kerschensteiner et al 1999; Chen et al 2003). GA-specific Th2 and Th1 cells both can secrete BDNF (Kipnis et al 2000; Ziemssen et al
2002) as well as other neurotrophic factors although Th2 cells seem to secrete higher levels of these factors than Th1 cells. In addition both MBP- and GA-reactive T cells make nerve growth factor (NGF) and neurotrophin 4/5 (NT-4/5) (Kipnis et al 2000). It is of interest that in both GA treated animals and naïve animals that receive GA reactive lymphocytes, neuroprotection can be demonstrated in both EAE (Aharoni et al 2000) as well as in models of optic nerve trauma (Kipnis et al 2000), retinal cell damage from glaucoma (Bakalash et al 2005) and degenerative diseases such as amyotrophic lateral sclerosis (Angelov et al 2003) and Parkinson's disease (Benner et al 2004). More recently, glatiramer has shown hints of efficacy in a mouse model of Alzheimer's disease (Frenkel et al 2005), as well as some neuroprotectant effect in organophosphate neurotoxicity (Schori et al 2005). However, whether the currently used regimen of GA in MS patients leads to the presence of BDNFsecreting $\mathrm{T}$ cells in the brain is unknown.

\section{Conclusion}

Glatiramer acetate is a drug with beneficial effects on the course of multiple sclerosis, reducing both relapse frequency and probably accumulation of deficit over time. The drug also has beneficial effects on MRI disease activity. Although the mechanisms of action are not known in detail, it is clear that they are multifactorial. There is a down-regulation of inflammatory activity in the MS brain, as well as a neuroprotectant effect. In fact, the elaboration of BDNF by $T$ cells may be useful in other disease states in which neurodegeneration plays a role.

\section{References}

Abramsky O, Teitelbaum D, Arnon R. 1977. Effect of a synthetic polypeptide (Cop 1) on patients with multiple sclerosis and with acute disseminated encephalomyelitis. J Neurol Sci, 31:433-8.

Aharoni R, Teitelbaum D, Arnon R, et al. 1999. Copolymer 1 acts against the immunodominant epitope $82-100$ of myelin basic protein by $\mathrm{T}$ cell receptor antagonism in addition to major histocompatibility complex blocking. Proc Nat Acad Sci US A, 96:634-9.

Aharoni R, Teitelbaum D, Leitner O, et al. 2000. Specific Th2 cells accumulate in the central nervous system of mice protected against experimental autoimmune encephalomyelitis by copolymer 1. Proc Nat Acad Sci U S A, 97:11472-7.

Aharoni R, Teitelbaum D, Sela M, et al. 1997. Copolymer 1 induces $\mathrm{T}$ cells of the T helper type 2 that crossreact with myelin basic protein and suppress experimental autoimmune encephalomyelitis. Proc Nat Acad Sci U S A, 94:10821-6.

Angelov D, Waibel S, Guntinas-Lichius O, et al. 2003. Therapeutic vaccine for acute and chronic motor neuron diseases: implications for ALS. Proc Nat Acad Sci US A, 100:4790-5.

Arnold D, De Stefano N, Narayan S, et al. 2001. Axonal injury and disability in multiple sclerosis: magnetic resonance spectroscopy as a measure of dynamic pathological change in white matter. In Filippi M, Arnold D, Comi G (eds). Magnetic resonance spectroscopy in multiple sclerosis. New York: Springer Verlag. p 61-7. 
Arnon R. 1996. The development of Cop 1 (Copaxone), an innovative drug for the treatment of multiple sclerosis: personal reflections. Immunol Lett, 50:1-15.

Bakalash S, Schlomo G, Aloni E, et al. 2005. T cell-based vaccination for morphological and functional neuroprotection in a rat model of chronically elevated intraocular pressure. J Mol Med, 83:904-16.

Balabanov R, Lisak D, Beaumont T, et al. 2001. Expression of urokinase plasminogen activator receptor on monocytes from patients with relapsing-remitting multiple sclerosis: effect of glatiramer acetate (copolymer 1). Clin Diagn Lab Immunol, 8:1196-203.

Benner E, Mosley R, Destache C, et al. 2004. Therapeutic immunization protects dopaminergic neurons in a mouse model of Parkinson's disease. Proc Nat Acad Sci US A, 101:9435-40.

Boneschi F, Rovaris M, Johnson K, et al. 2003. Effects of glatiramer acetate on relapse rate and accumulated disability in multiple sclerosis: metaanalysis of three double-blind, randomized, placebo-controlled clinical trials. Mult Scler, 9:349-55.

Bornstein M, Miller A, Slagle S, et al. 1987. A pilot trial of Cop 1 in exacerbating-remitting multiple sclerosis. New Engl J Med, 317:408-14.

Bornstein M, Miller A, Slagle S, et al. 1991. A placebo-controlled, doubleblind, randomized, two-center, pilot trial of Cop 1 in chronic progressive multiple sclerosis. Neurology, 41:533-9.

Bornstein M, Miller A, Teitelbaum D, et al. 1981. Multiple sclerosis: trial of a synthetic polypeptide. Ann Neurol, 11:317-19.

Brenner T, Arnon R, Sela M, et al. 2001. Humoral and cellular immune responses to Copolymer 1 in multiple sclerosis patients treated with Copaxone. J Neuroimmunol, 115:152-60.

Burns J, Littlefield K. 1991. Failure of copolymer 1 to inhibit the human T cell response to myelin basic protein. Neurology, 41:1317-19.

Caramanos Z, Arnold D. 2005. Evidence for use of glatiramer acetate in multiple sclerosis. Lancet Neurol, 4:74-5.

Chen M, Gran B, Costello K, et al. 2001. Glatiramer acetate induces a Th2-biased response and crossreactivity with myelin basic protein in patients with MS. Mult Scler, 7:209-19.

Chen M, Valenzuela R, Dhib-Jalbut S. 2003. Glatiramer acetate-reactive T cells produce brain-derived neurotrophic factor. J Neurol Sci, 215:37-44.

Comi G, Filippi M, Wolinsky J, et al. 2001. European/Canadian multicenter, double-blind, randomized, placebo-controlled study of the effects of glatiramer acetate on magnetic resonance imaging-measured disease activity and burden in patients with relapsing multiple sclerosis. Ann Neurol, 49:290-7.

Comi G, Hartung H-P, Boneschi F. 2005. Evidence for use of glatiramer acetate in multiple sclerosis. Lancet Neurol, 4:75-6.

Duda P, Schmied M, Cook S, et al. 2000. Glatiramer acetate induces degenerate, Th2-polarized immune responses in patients with multiple sclerosis. J Clin Invest, 105:967-76.

Farina C, Bergh F, Abrecht H, et al. 2001. Treatment of multiple sclerosis with Copaxone (COP). Elispot assay detects COP-induced interleukin-4 and interferon-gamma response in blood cells. Brain, 124:705-19.

Farina C, Vargas V, Heydari N, et al. 2002. Treatment with glatiramer acetate induces specific IgG4 antibodies in multiple sclerosis patients. J Neuroimmunol, 123:188-92.

Farina C, Weber M, Meinl E, et al. 2005. Glatiramer acetate in multiple sclerosis: an update on potential mechanisms of action. Lancet Neurol, 4:567-75

Filippi M, Rovaris M, Rocca M, et al. 2001. Glatiramer acetate reduces the proportion of new MS lesions evolving into "black holes." Neurology, 57:731-3.

Fridkis-Hareli M, Teitelbaum D, Gurevich E, et al. 1994. Direct binding of myelin basic protein and synthetic copolymer 1 to class II major histocompatibility complex molecules on living antigen-presenting cells - specificity and promiscuity. Proc Nat Acad Sci US A, 91:4872-6.

Ge Y, Grossman R, Udupa J, et al. 2000. Glatiramer acetate (Copaxone) treatment in relapsing-remitting MS. Quantitative MR assessment. Neurology, 54:813-17.
Hussien Y, Sanna A, Soderstrom M, et al. 2001. Glatiramer acetate and IFN-beta act on dendritic cells in multiple sclerosis. J Neuroimmunol, 121:102-10.

Johnson K, Brooks B, Cohen J, et al. 1998. Extended use of glatiramer acetate (Copaxone) is well tolerated and maintains its clinical effect on multiple sclerosis relapse rate and degree of disability. Neurology, 50:701-8.

Johnson K, Brooks B, Cohen J, et al. 1995. Copolymer 1 reduces relapse rate and improves disability in relapsing-remitting multiple sclerosis: results of a phase III multicenter, double-blind, placebo-controlled trial. Neurology, 45:1268-76.

Johnson K, Ford C, Lisak R, et al. 2005. Neurologic consequence of delaying glatiramer acetate therapy for multiple sclerosis: 8 year data. Acta Neurol Scand, 111:42-7.

Kalinski P, Hilkens C, Wierenga E, et al. 1999. T cell priming by type-1 and type-2 polarized dendritic cells: the concept of a third signal. Immunol Today, 20:561-7.

Karandikar N, Crawford M, Yan X, et al. 2002. Glatiramer acetate (copaxone) therapy induces CD8 $\mathrm{T}$ cell responses in patients with multiple sclerosis. J Clin Invest, 109:641-9.

Kerschensteiner M, Gallmeier E, Behrens L, et al. 1999. Activated human $\mathrm{T}$ cells, B cells, and monocytes produce brain-derived neurotrophic factor in vitro and in inflammatory brain lesions: a neuroprotective role of inflammation. J Exp Med, 189:865-70.

Khan O, Shen Y, Caon C, et al. 2005. Axonal metabolic recovery and potential neuroprotective effect of glatiramer acetate in relapsing-remitting multiple sclerosis. Mult Scler, 11:646-51.

Kidd D, Barkhof R, McConnell R, et al. 1999. Cortical lesions in multiple sclerosis. Brain, 122:17-26.

Kim H, Ifergan I, Antel J, et al. 2004. Type 2 monocyte and microglia differentiation mediated by glatiramer acetate therapy in patients with multiple sclerosis. J Immunol, 172:7144-53.

Kipnis J, Yoles E, Porat Z, et al. 2000. T cell immunity to copolymer 1 confers neuroprotection on the damaged optic nerve: possible therapy for optic neuropathies. Proc Nat Acad Sci US A, 97:7446-51.

Kurtzke J. 1983. Rating neurologic impairment in multiple sclerosis: an expanded disability status scale (EDSS). Neurology, 33:1444-52.

Kutzelnigg A, Lucchinetti C, Stadelmann C, et al. 2005. Cortical demyelination and diffuse white matter injury in multiple sclerosis. Brain, 128:2705-12.

Lisak R, Zweiman B, Blanchard N, et al. 1983. Effect of treatment with copolymer 1 (Cop-1) on the in vivo and in vitro manifestations of experimental allergic encephalomyelitis (EAE). J Neurol Sci, 62:281-93.

Lucchinetti C, Bruck W, Parisi J, et al. 2000. Heterogeneity of multiple sclerosis lesions: implications for the pathogenesis of demyelination. Ann Neurol, 47:707-17.

Munari L, Lovati R, Boiko A. 2003. Therapy with glatiramer acetate for multiple sclerosis (review). The Cochrane Database of Systematic Reviews: CD004678.

Neuhaus O, Farina C, Yassouridis A, et al. 2000. Multiple sclerosis: comparison of copolymer-1-reactive $\mathrm{T}$ cell lines from treated and untreated subjects reveals cytokine shift from T helper 1 to T helper 2 cells. Proc Nat Acad Sci U S A, 97:7452-7.

Peterson J, Bo L, Mork S, et al. 2001. Transected neurites, apoptotic neurons, and reduced inflammation in cortical multiple sclerosis lesions. Ann Neurol, 50:389-400.

Qin Y, Zhang D, Prat A, et al. 2000. Characterization of T cell lines derived from glatiramer-acetate-treated multiple sclerosis patients. J Neuroimmunol, 108:201-6.

Ragheb S, Abramczyk S, Lisak D, et al. 2001. Long-term therapy with glatiramer acetate in multiple sclerosis: effect on T cells. Mult Scler, 7:43-7.

Raine C, Cannella B, Hauser S, et al. 1999. Demyelination in primate autoimmune encephalomyelitis and acute multiple sclerosis lesions: a case for antigen-specific antibody mediation. Ann Neurol, 46:144-60 
Rivers T, Schwentker F. 1935. Encephalomyelitis accompanied by myelin destruction experimentally produced in monkeys. $J$ Exp Med, 61:689-702.

Salama H, Hong J, Zang Y, et al. 2003. Blocking effects of serum reactive antibodies induced by glatiramer acetate treatment in multiple sclerosis. Brain, 126:2638-47.

Sormani M, Rovaris M, Valsasina P, et al. 2004. Measurement error of two different techniques for brain atrophy assessment in multiple sclerosis. Neurology, 62:1432-4.

Teitelbaum D, Brenner T, Abramsky O, et al. 2003. Antibodies to glatiramer acetate do not interfere with its biological functions and therapeutic efficacy. Mult Scler, 9:592-9.

Teitelbaum D, Fridkis-Hareli M, Arnon R, et al. 1996. Copolymer 1 inhibits chronic relapsing experimental aller/gic encephalomyelitis induced by proteolipid protein (PLP) peptides in mice and interferes with PLPspecific T cell responses. J Neuroimmunol, 64:209-17.

Teitelbaum D, Milo R, Arnon R, et al. 1992. Synthetic copolymer 1 inhibits human T-cell lines specific for myelin basic protein. Proc Nat Acad Sci USA, 89:137-41.
Tselis A, Khan O. 2004. Multiple sclerosis. A case for early treatment. In Olek M (ed). Multiple sclerosis. biology, diagnosis and new treatment strategies. Totowa, NJ: Human Press. p 131-43.

Vieira P, Heystek H, Wormmeester J, et al. 2003. Glatiramer acetate promotes Th2 cell development and increased IL-10 productiion through modulation of dendritic cells. J Immunol, 170:4483-8.

Weber M, Starck M, Wagenpfeil S, et al. 2004. Multiple sclerosis: glatiramer acetate inhibits monocyte reactivity in vitro and in vivo. Brain, 127:1370-8.

Wolinsky J. 2004. The PROMiSe trial: baseline data review and progress report. Mult Scler, 10:S65-S72.

Wolinsky J, Comi G, Filippi M, et al. 2002. Copaxone's effect on MRImonitored disease in relapsing MS is reproducible and sustained. Neurology, 59:1284-6.

Ziemssen T, Kumpfel T, Klinkert W, et al. 2002. Glatiramer acetatespecific T-helper-1 and 2-type cell lines produce BDNF: implications for multiple sclerosis therapy. Brain, 125:2381-91. 
